\title{
Potential problems with interpolating fields
}

\author{
Michael C. Birse ${ }^{\mathrm{a}}$ \\ Theoretical Physics Division, School of Physics and Astronomy, The University of Manchester, Manchester, M13 9PL, UK
}

Received: 8 September 2017

Published online: 24 November 2017

(c) The Author(s) 2017. This article is published with open access at Springerlink.com

Communicated by S. Hands

\begin{abstract}
A potential can have features that do not reflect the dynamics of the system it describes but rather arise from the choice of interpolating fields used to define it. This is illustrated using a toy model of scattering with two coupled channels. A Bethe-Salpeter amplitude is constructed which is a mixture of the waves in the two channels. The potential derived from this has a strong repulsive core, which arises from the admixture of the closed channel in the wave function and not from the dynamics of the model.
\end{abstract}

\section{Introduction}

In their work on two-baryon systems in lattice QCD, members of the HAL-QCD collaboration have extracted baryon-baryon potentials from their simulations [1-7]. The method relies on the construction of a Bethe-Salpeter amplitude or "wave function" - a matrix element of two interpolating fields with the quantum numbers of the baryons involved. This amplitude is then inserted into a Schrödinger equation in order to deduce the baryonbaryon potential that would generate it. More recently, the same idea has been used to extract short-distance potentials through the operator product expansion of perturbative QCD [8-11]. A review of the approach can be found in ref. [12].

One interesting feature of the resulting nucleonnucleon potential is that it possesses a repulsive core [1], very reminiscent of those in many of the potentials traditionally used in nuclear physics, such as Argonne $v_{18}$ [13]. When extended to three flavours of quark with $S U(3)$ symmetry, repulsive cores are seen in all channels except the $S U(3)$ singlet [4]. The repulsion is particularly strong in the symmetric octet channel, where no long-range attraction is seen.

This pattern is qualitatively similar to that seen in constituent quark models (for reviews, see refs. [14-16]). For two octet baryons, the symmetric octet channel is forbidden by the Pauli principle at small separations, leading to strong repulsion. Other channels are not forbidden and so, as well as antisymmetrisation, the form of the interaction between the quarks is needed, as discussed by Oka [17]. In quark models based on magnetic gluon exchange, short-distance repulsion is found in all channels except the singlet.

\footnotetext{
a e-mail: mike.birse@manchester.ac.uk
}

The HAL-QCD approach has been criticised, for example in refs. $[18,19]$, because a potential is not an observable in either experiments or lattice simulations. In the lattice case, the energies of two-baryon states are observables and these can be related, via Lüscher's formula [20], to experimental observables, namely, phase shifts. In the HALQCD approach, this information on the physical phase shifts is encoded in the tails of the Bethe-Salpeter amplitudes outside the interaction region. In contrast, the short-distance forms of these amplitudes, and hence the deduced potentials are dependent on the choice of interpolating field used to define them.

More recently, the HAL-QCD collaboration have noted that the times at which they determine their potentials may not be sufficiently large for a single state to dominate the correlator used to extract the Bethe-Salpeter amplitude. They have therefore introduced a version of their approach based on a time-dependent Schrödinger equation [7,21-23]. A potential issue with this is that, since a number of energy eigenstates contribute to the correlator, there is no single phase shift determining the form of Bethe-Salpeter amplitude at large separations. It is thus not obvious that the potentials determined from these amplitudes are "anchored" to physical observables.

In this short note, I use a toy model of scattering with two coupled channels to illustrate how a potential constructed in this way can develop features that reflect the choice of interpolating fields, and not the actual dynamics. In particular an admixture of a closed channel can lead to a strong repulsive core in the derived potential that does not correspond to any aspect of the potentials in the original model. Finally, I comment on how this model may be able to check whether the time-dependent version of the HAL QCD approach leads to potentials that reproduce the correct phase shifts. 


\section{Coupled-channel model}

The toy model I use to illustrate issues with interpreting potentials from Bethe-Salpeter wave functions is two-body scattering in one dimension with two coupled channels. The lower-energy channel can be thought of as corresponding to two nucleons in their ground states; the other to one ground-state nucleon and one $\mathrm{N}^{*}$ with the same quantum numbers. I shall refer to the channels as "NN" and "NN"". The particles in the NN channel interact through an attractive square-well potential similar to the long-range attractive force between nucleons. For simplicity I take the potential in the $\mathrm{NN}^{*}$ channel to be zero. The $\mathrm{N}^{*}$ has an excitation energy $\Delta$ and so the $\mathrm{NN}^{*}$ channel is closed for energies below $\Delta$. The two channels are coupled by a contact interaction of strength $g$.

While this model is very much a caricature of real nucleon-nucleon scattering, I believe that it retains enough aspects of the real system to illustrate why one should be wary about taking seriously short-distance features.

The wave functions for the relative motion of the particles in the two channels, $\psi_{0}(x)$ and $\psi_{1}(x)$, satisfy the coupled equations,

$$
\begin{gathered}
{\left[-\frac{1}{M} \frac{\mathrm{d}^{2}}{\mathrm{~d} x^{2}}+V(x)-E\right] \psi_{0}(x)+2 g \delta(x) \psi_{1}(x)=0} \\
{\left[-\frac{1}{M} \frac{\mathrm{d}^{2}}{\mathrm{~d} x^{2}}+\Delta-E\right] \psi_{1}(x)+2 g \delta(x) \psi_{0}(x)=0}
\end{gathered}
$$

where

$$
V(x)= \begin{cases}-V_{0} & \text { for }|x|<a \\ 0 & \text { for }|x|>a\end{cases}
$$

A general interpolating field for the "nucleon" in this model is just a linear combination of the $\mathrm{N}$ and $\mathrm{N}^{*}$ fields. A Bethe-Salpeter amplitude $\Psi(x)$ can be defined for any coupled-channel solution by first building a secondquantised state representing the solution and then taking the matrix element of a pair of interpolating field operators between this state and the vacuum. The result is just a linear combination of the two wave functions,

$$
\Psi(x)=\psi_{0}(x)+p \psi_{1}(x) .
$$

Since it is not required for the determination of the potential, I have not specified the overall normalisation of this quantity; only the coefficient $p$ of the $\mathrm{N}^{*}$ admixture is relevant.

The (even-parity) solutions of the coupled equations (2) have the forms

$$
\begin{aligned}
& \psi_{0}(x)= \begin{cases}A \cos (K|x|)+B \sin (K|x|) & \text { for }|x|<a \\
C \cos (k|x|)+D \sin (k|x|) & \text { for }|x|>a\end{cases} \\
& \psi_{1}(x)=\exp (-\alpha|x|),
\end{aligned}
$$

where

$$
\begin{aligned}
k^{2} & =M E, \\
K^{2} & =M\left(E+V_{0}\right), \\
\alpha^{2} & =M(\Delta-E) .
\end{aligned}
$$

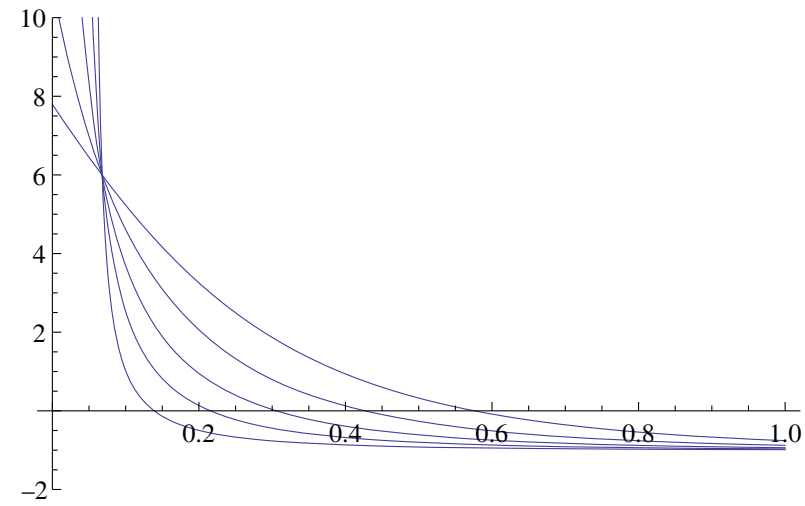

Fig. 1. The zero-energy potential $M V_{B S}(x ; 0)$ for $p=0.1$ (narrowest "core"), 0.25, 0.5, 1, 2 (widest).

Again the overall normalisation is irrelevant, and so I have arbitrarily taken $\psi_{1}(0)=1$.

Matching the solutions at the edge of the square well and at the origin, where the $\delta$-function acts, leads to the boundary conditions,

$$
\begin{aligned}
\psi_{0}(a-) & =\psi_{0}(a+), \\
\psi_{0}^{\prime}(a-) & =\psi_{0}^{\prime}(a+), \\
\psi_{0}^{\prime}(0) & =M g \psi_{1}(0), \\
\psi_{1}^{\prime}(0) & =M g \psi_{0}(0) .
\end{aligned}
$$

These give rise to a set of linear equations for the coefficients, $A, B, C, D$ :

$$
A \cos (K a)+B \sin (K a)=C \cos (k a)+D \sin (k a),
$$

$-K A \sin (K a)+K B \cos (K a)=-k C \sin (k a)+k D \cos (k a)$,

$$
\begin{aligned}
K B & =M g, \\
-\alpha & =M g A,
\end{aligned}
$$

which can be solved straightforwardly. The resulting wave functions at energy $E$ will be denoted $\psi_{0,1}(x ; E)$. From these, we can construct the Bethe-Salpeter amplitude $\Psi(x ; E)$.

Following the approach outlined in the introduction, I now use $\Psi(x ; E)$ to define a potential by demanding that it satisfy the Schrödinger equation,

$$
\left[-\frac{1}{M} \frac{\mathrm{d}^{2}}{\mathrm{~d} x^{2}}+V_{B S}(x ; E)-E\right] \Psi(x ; E)=0 .
$$

The resulting potential is

$$
V_{B S}(x ; E)=\frac{1}{M \Psi(x ; E)} \frac{\mathrm{d}^{2} \Psi}{\mathrm{d} x^{2}}+E .
$$

\section{Results}

I present results here for a representative parameter set, $M V_{0}=1 / a^{2}, M \Delta=6 / a^{2}$ and $M g=6 / a$, and I take $a=1$ for convenience in plotting the results. Figure 1 


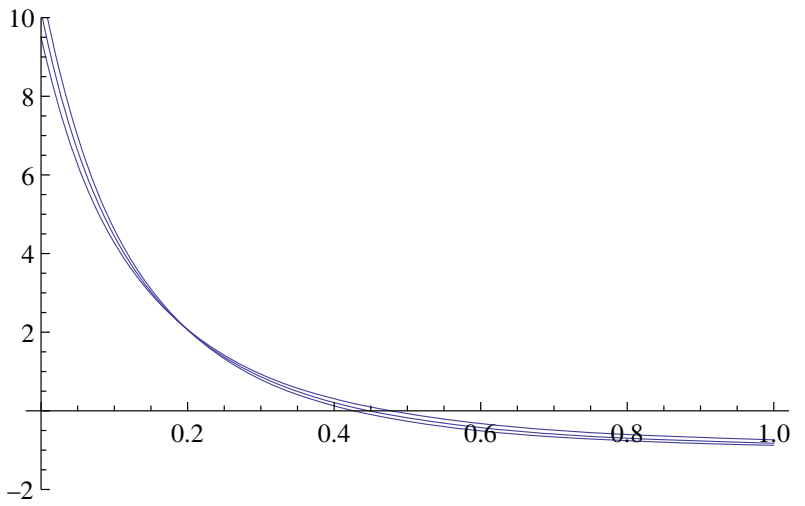

Fig. 2. The potential $M V_{B S}(x ; E)$ for $M E=0,1,2$, all with $p=1$.

shows the zero-energy potential $V_{B S}(x ; 0)$ for a range of values of the mixing parameter in the interpolating field. Note that I have not plotted the potential for $x>a$, where it is essentially zero, nor have I shown the $\delta$ function that is present at the origin.

A couple of features are worth noting about these potentials. First, the long-range attraction in the NN channel, $V(x)=-V_{0}$, can be seen as $x$ approaches 1 . This similar to the way that the pion-exchange tail can be seen in the potentials extracted by the HAL QCD Collaboration [1]. The second feature is the apparent repulsive "hard core" that is present in all cases for $x \lesssim 0.5$. This comes entirely from the closed $\mathrm{NN}^{*}$ channel, which makes a large positive contribution to $V_{B S}(x ; 0)$ for $x \lesssim 1 / \alpha$ because $\mathrm{d}^{2} \psi_{1}(x) / \mathrm{d} x^{2} \simeq M \Delta \psi_{1}(x)$ there. It has nothing to do with the NN-channel wave function, as demonstrated by its strong dependence on the choice of interpolating field. In contrast, the long-range attraction (where it is not swamped by the core) is independent of the choice of field.

At low energies, the core in this potential is only weakly dependent on the energy $E$, as can be seen in fig. 2 . Like the HAL-QCD potentials, this has the appearance of something that could be well approximated by a local potential. However this is just a reflection of the fact that the strength and range of the core arise from the closedchannel wave function $\psi_{1}(x)$, and so are controlled by $g$ and $\Delta$ (and $p$ of course), so long as $E$ lies well below the excitation energy $\Delta$.

\section{Conclusion}

The results in the previous section illustrate how a potential can have features that arise from the interpolating fields used to define it. In particular they show how coupling to closed channels can lead to repulsive cores in potentials derived from Bethe-Salpeter amplitudes. Similar repulsive cores seen in the HAL-QCD collaboration's analyses of their lattice simulations [1-7] could therefore be results of their choice of interpolating fields, rather than indications of similar dynamical mechanisms to those of the constituent quark model.

Since the same interpolating fields are used in all channels, one might argue that the absence of a repulsive core in the $S U(3)$-singlet channel is evidence against these cores being artefacts of the choice of field. However the strength of the core is dependent on the size of the coupling to the closed channel and, for baryons in the $S U(3)$ limit, this coupling is likely to be strongly channel-dependent. In particular, this dependence is to be expected if the coupling to excited octet baryon states occurs via intermediate excitation of decuplet baryons. Such excitations could be mediated by pion and kaon exchanges at long distances or, at shorter ranges, by the same magnetic gluon interactions that are responsible for the repulsive cores in the quark model [17]. This mechanism for generating excited octet baryons would not operate in the $S U(3)$-singlet channel since the relevant intermediate states - octet-decuplet or decuplet-decuplet - cannot couple to an overall singlet. It could therefore explain the absence of a repulsive core in that channel.

To summarise: a potential is not an observable. The toy model in this note provides a warning that even quite striking features of a potential may not reflect the actual dynamics of the system it describes.

A final comment is that this toy model provides a very simple setting where Bethe-Salpeter amplitudes can be constructed with known phase shifts. It can therefore help to check the extent to which potentials extracted from these amplitudes do reproduce phase shifts. Indeed the model has already been used by Sugiura et al. to study the convergence of a derivative expansion of energyindependent but nonlocal potentials [24]. As noted in the introduction, the time-dependent version of the HAL QCD approach uses correlators that are not dominated by a single energy eigenstate and so do not lead to BetheSalpeter amplitudes with well-defined phase shifts. This is an issue for local potentials determined by this approach, although not necessarily for nonlocal ones. An extension of the model studies in ref. [24] to time-dependent amplitudes could shed light on how to implement the method in a way that reproduces physical phase shifts [25].

I am grateful to T. Sugiura and N. Ishii for discussions of how this model could be used to test their approach. This work was supported by the UK STFC under grants ST/J000159/1, ST/L005794/1 and ST/P004423/1.

Open Access This is an open access article distributed under the terms of the Creative Commons Attribution License (http://creativecommons.org/licenses/by/4.0), which permits unrestricted use, distribution, and reproduction in any medium, provided the original work is properly cited. 


\section{References}

1. N. Ishii, S. Aoki, T. Hatsuda, Phys. Rev. Lett. 99, 022001 (2007).

2. H. Nemura, N. Ishii, S. Aoki, T. Hatsuda, Phys. Lett. B 673, 136 (2009).

3. S. Aoki, T. Hatsuda, N. Ishii, Prog. Theor. Phys. 123, 89 (2010).

4. T. Inoue et al., Prog. Theor. Phys. 124, 591 (2010).

5. K. Murano, N. Ishii, S. Aoki, T. Hatsuda, Prog. Theor. Phys. 125, 1225 (2011).

6. T. Doi, Prog. Theor. Phys. 127, 723 (2012).

7. T. Inoue et al., Phys. Rev. Lett. 106, 162002 (2011).

8. S. Aoki, J. Balog, P. Weisz, JHEP 05, 008 (2010).

9. S. Aoki, J. Balog, P. Weisz, JHEP 09, 083 (2010).

10. S. Aoki, J. Balog, P. Weisz, New J. Phys. 14, 043046 (2012).

11. S. Aoki, J. Balog, P. Weisz, Prog. Theor. Phys. 128, 1269 (2012).

12. S. Aoki, Prog. Part. Nucl. Phys. 66, 687 (2011).
13. R.B. Wiringa, V.G.J. Stoks, R. Schiavilla, Phys. Rev. C 51, 38 (1995).

14. M. Oka, K. Yazaki, in Quarks and Nuclei, edited by W. Weise (World Scientific, Singapore, 1984) p. 489.

15. M. Oka, K. Shimizu, K. Yazaki, Prog. Theor. Phys. Suppl. 137, 1 (2000).

16. Y. Fujiwara, Y. Suzuki, C. Nakamoto, Prog. Part. Nucl. Phys. 58, 439 (2007).

17. M. Oka, Nucl. Phys. A 881, 6 (2012).

18. W. Detmold, K. Orginos, M.J. Savage, Phys. Rev. D 76, 114503 (2007).

19. S.R. Beane, K. Orginos, M.J. Savage, Int. J. Mod. Phys. E 17, 1157 (2008).

20. M. Lüscher, Nucl. Phys. B 354, 531 (1991).

21. T. Inoue et al., Nucl. Phys. A 881, 28 (2012).

22. N. Ishii et al., Phys. Lett. B 712, 437 (2012).

23. T. Kurth, N. Ishii, T. Doi, S. Aoki, T. Hatsuda, JHEP 12, 015 (2013).

24. T. Sugiura, N. Ishii, M. Oka, Phys. Rev. D 95, 074514 (2017).

25. N. Ishii, private communication. 